\title{
A small-animal inhalation chamber for short-to-intermediate term exposure
}

\author{
W. KEITH PRUSACZYK and GLORIA J. FISCHER \\ Washington State University, Pullman, Washington
}

\begin{abstract}
An inhalation chamber for behavioral toxicology studies is described. Complete and uniform diffusion of test atmospheres throughout the chamber is achieved, thus solving a dead-airspace problem prevalent in extant chamber designs. Modular attachments to the chamber allow for a variety of uses, including physiological-pathological and classical or operant conditioning. The chamber design and size are appropriate for testing most small laboratory animals (e.g., birds, rodents, and small primates), but the chamber is easily modified for use with larger vertebrates. Despite the versatility of design, the chamber is relatively inexpensive and easy to build.
\end{abstract}

Typically, atmospheric inhalation studies are performed in whole-body chambers to assess morphological or physiological effects of breathing toxic substances (MacFarland, 1976). Such chambers have been designed either for chronic (i.e., days to years) exposure to a toxic substance (e.g., Leong, Powell, Pochyla, \& Lummis, 1981; MacFarland, Ulrich, Martin, Krumm, Busey, \& Alarie, 1971; Moss, 1981; Rosenbaum, Makoff, \& Mills, $1969)$ or for acute ( $\leqslant 5-\mathrm{min})$ exposure (e.g., Koblin, Dong, \& Eger, 1979). Less common are chambers designed for intermediate (i.e., hours) exposure, which usually are used to assess the effects of inhaling mildly toxic or nontoxic atmospheres (e.g., nitrous oxide) on behavior (e.g., Goudie \& Dickens, 1978; Wallenstein, 1976).

There are basic requirements of any whole-body inhalation chamber. The first is internal atmospheric integrity. Thus, it must be possible to seal the chamber hermetically. Second, there should be little or no leakage, especially of a toxic test substance. To achieve this, the chamber should have a slightly negative internal pressure or be sufficiently sturdy to withstand elevated flow rates and/or atmospheric pressure. Third, the chamber should be resistant to caustic actions of the test substance. If this basic whole-body chamber is to be used for an intermediate exposure duration, then an additional requirement is the complete diffusion of the test atmosphere throughout the chamber to eliminate areas of low substance turnover, or so-called "deadspots." This problem has not been addressed adequately in the design of any of the chambers considered above. All ap-

The design and construction of this apparatus were supported by Grant NR 201459 from the office of Naval Research, under the direction of the junior author. The authors wish to thank Tom Hellesto, who contributed to the design and directed the construction of the chamber. Thanks also are due Barbara J. Vail and Bryan Kulak, who used and tested the chambers in a CTA experiment supported by the same ONR grant. Blue prints for constructing the chamber are available on request. The authors' mailing address is: Department of Psychology, Washington State University, Pullman, Washington 99164. pear to be inadequate in maintaining complete diffusion of the test gas, due primarily to the use of single testsubstance input and exhaust vents. With input through a single vent, the atmosphere tends to "stream" (i.e., flow is relatively direct between input and exhaust, creating a football-shaped flow pattern with apexes at the vents). This stream tends to produce eddying currents in surrounding areas, such that turnover of the test substance in those areas is lower than the turnover in the main stream.

Areas of low turnover, or dead spots, usually are not of concern in acute exposure experiments, because exposure duration is brief and pathological changes are usually substantial. Dead spots are of little concern in chronic exposure experiments, because an animal exposed for long periods moves about the chamber, thereby receiving a more uniform exposure over time. Or, more often, several animals are exposed simultaneously, in individual cages placed inside the chamber. In such cases, the problem of dead spots has been redressed by installation of a baffle system that induces circular airflows (Moss, 1981) or, more directly, by using a wideangle air-pressure nozzle to widen the stream and then placing cages within the stream (Leong et al., 1981).

Although adequate for chronicexposure studies, placing a cage within a widened stream, inside an inhalation chamber, is not applicable to intermediate exposure duration. Not only would the larger chamber be costly to build, but also a substantial portion of any shortto-intermediate exposure time would be used up in dispersing the test substance sufficiently to achieve uniform flow. Instead, with short-to-intermediate exposure, especially in behavioral studies, the animal needs to move about freely in a chamber appropriate to its size. Dead spots can produce significant problems under these conditions. For example, rats, at least, appear attracted to areas of low turnover (Moss, Note 1). Attraction to these areas creates the potential for an additional problem, the accumulation of metabolic wastes. The resulting increase in nitrogenous waste gases might be 
inadequately exhausted from areas of low atmospheric turnover.

The present chamber was designed for use in behavioral studies to overcome the problem of lower atmospheric turnover in certain areas, that is, in dead spots.

\section{DESIGN OF THE CHAMBER}

\section{Gas Diffusion}

The method used to overcome the problem of dead spots involves a dual diffusion system that insures uniform diffusion of a test gas throughout the entire animal enclosure area. An initial gross diffusion is accomplished by reversing the gas input port so that the gas passing through the opening is directed back toward the wall through which it has entered. As can be seen in Figure 1, there are four such input ports on each of two opposing walls of the chamber. As the stream of gas strikes the wall, it is initially diffused along the wall. A subsequent, finer diffusion is accomplished by a secondary screen, a multiholed Plexiglas plate, placed $1.25 \mathrm{~cm}$ from the input ports and forming one wall of the animal enclosure area (see Figure 1). Each of the large number of small $(1.585-\mathrm{mm})$ holes in the secondary diffuser acts as a secondary source of the test gas. Since the holes are distributed $6.35 \mathrm{~mm}$ apart throughout the Plexiglas screen in the wall of the enclosure area, the system promotes an even diffusion of gas to all areas the animal might seek out. An identical dual system is used as the exhaust system, to prevent the formation of streams of gas at the exhaust ports. There, each hole acts as an individual exhaust port. The large number of these exhaust ports prevents streaming.

The adequacy of the diffusion system was tested with $\mathrm{CO}_{2}$ and smoke-capsule infusion. In both cases, the substances were observed to diffuse completely and continuously throughout the animal enclosure area.

\section{Animal Loading}

MacFarland (1976) suggested that an animal should occupy no more than $5 \%$ of the volume of the atmospheric containment area. As a margin of safety, the present chamber was designed to operate at $3 \%$ to $4 \%$ animal loading. Given an approximate density of mammalian tissue of about $1 \mathrm{~g} / \mathrm{cm}^{2}$ and an upper weight limit of about $500 \mathrm{~g}$ for an adult rat, the chamber size was cal-

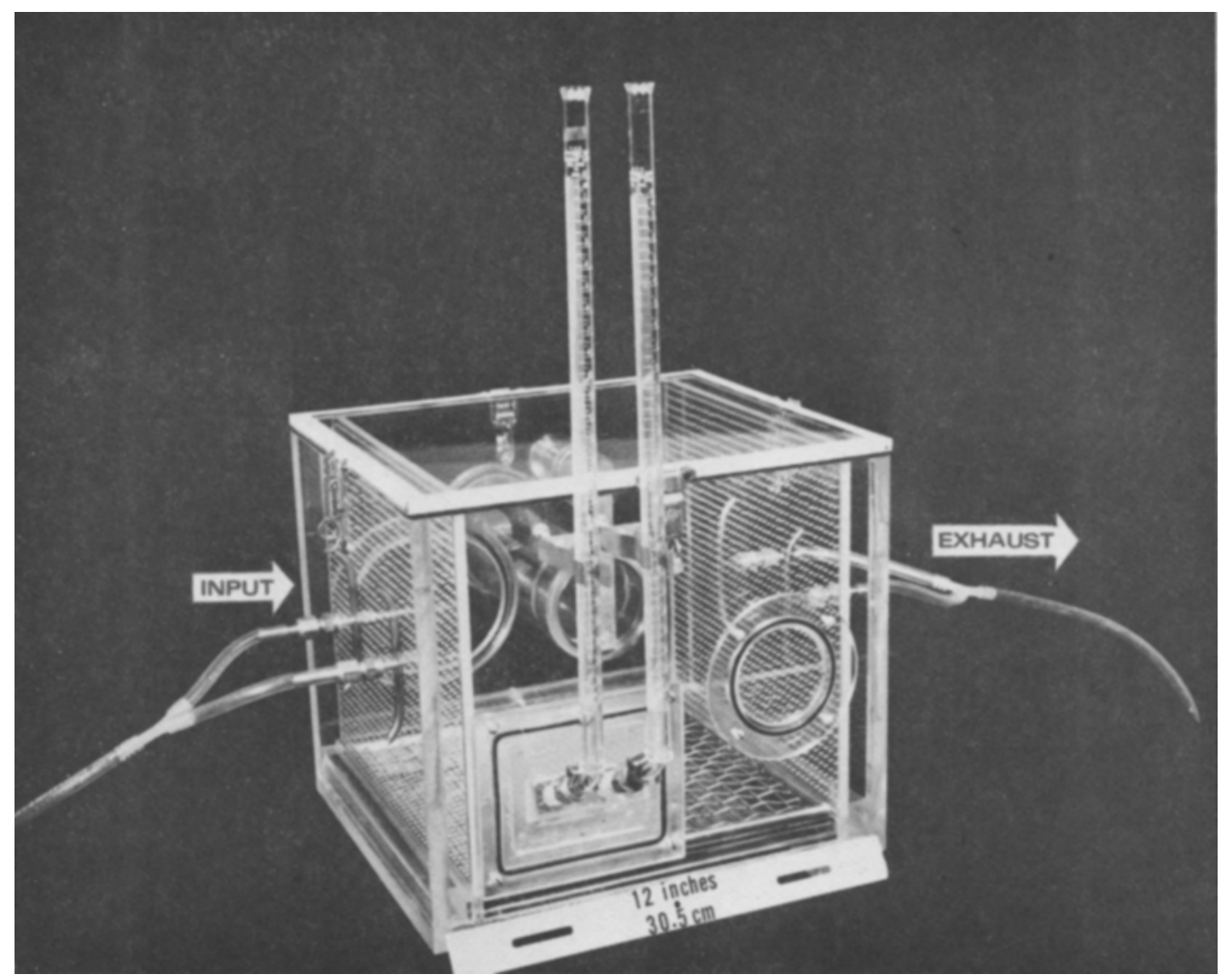

Figure 1. Rear view of inhalation chamber, showing flow direction and the conditioned taste aversion (drinking tube) module. 
culated to a $25 \times 25 \times 22.5 \mathrm{~cm}$ (14.2-liter) containment area. Thus, the chamber operates well within a safe loading range for animals weighing $\leqslant 500 \mathrm{~g}$, but can handle animals weighing up to $700 \mathrm{~g}$ (at $5 \%$ loading). A flow rate in excess of 1 chamber volume per minute can produce a draft perceptible to the test animal (Moss, Note 1). As a result, a flow rate of 10 liters/min (as measured by a Manostat 36-541-22 flowmeter) yields a flow of less than 0.75 chamber volumes per minute.

\section{Metabolic Wastes}

When exposure occurs for a period of $1 \mathrm{~h}$ or less, metabolic contamination of a chamber by a large rodent appears to be of a level so low as to warrant no real concern (unless the animal loading is high, i.e., around or, especially, above 5\%). For exposure periods exceeding 1-2 h, however, control for waste materials, especially ammonia-generating substances, becomes desirable (Barrow \& Dodd, 1979). Accordingly, the present chamber incorporates controls for: (1) digestive waste materials (urine and feces) and ammonia gas generated by such wastes over exposure durations of $1-2 \mathrm{~h}$ and (2) elimination of $\mathrm{CO}_{2}$. To control the first, fecal wastes fall through a removable (for ease of cleaning) wiremesh floor into absorbent hardwood shavings in a metal tray below. Any ammonia gas accumulating from these wastes over 1-2-h exposure is removed in the normal internal atmospheric turnover. If, however, the test period is extended substantially beyond $2-3 \mathrm{~h}$, an opening on the front of the chamber is of a size $(2.54 \times 27 \mathrm{~cm})$ convenient for tray removal and replacement. Since gas is lost with removal of the tray in an amount proportional to opening size and duration of opening, a quick tray change could "clean" the chamber with only minimal disruption of the in. ternal atmosphere and allow rapid recovery of atmospheric integrity.

Respiratory waste $\left(\mathrm{CO}_{2}\right)$ is handled in two ways. First, the normal atmospheric turnover removes $\mathrm{CO}_{2}$ expired into the chamber. As an additional precaution, a $\mathrm{CO}_{2}$ absorbent (e.g., Sodasorb) that does not react with the test substance may be sprinkled among the hardwood shavings in the metal tray.

\section{Behavioral Attachments}

A second unique characteristic of the chamber is its adaptability for use in behavioral experimentation. Walsh and Bachrach (1974) placed a complete Harvardtype operant chamber inside a large hyperbaric chamber to study behavioral toxicology. This approach seems to be less appropriate to the study of dynamic atmospheres at normal pressure, since an enclosed operant apparatus interferes with the atmospheric integrity and the flow of test substances within the chamber. Also, there is the problem noted previously, the need to use a substantial portion of any short-to-intermediate exposure to disperse a gas adequately throughout the larger chamber. Most appropriate for behavioral assessment are components that are part of the chamber and do not disturb atmospheric integrity. This requirement is met with two modular component systems designed for use in studies of classical [e.g., a conditioned taste aversion (CTA) paradigm] or operant conditioning. In addition, shock avoidance conditioning could be added by replacing the wire-mesh floor with a shock grid.

The CTA (drinking) paradigm requires two accessible, calibrated drinking tubes. As can be seen in Figure 2, the tube module attaches vertically to the outside of the main chamber, to a wall not having a secondary diffusion screen. The tubes are attached to the exterior by phosphor-bronze spring-loaded tension clips. The stainless steel licking spouts of both tubes are mounted permanently at a 45-deg angle through a Plexiglas plate. The calibrated glass tubes are attached to the drinking spouts by a short section of nalgene tubing, for ease of removal. The Plexiglas plate attaches flush from the outside of the chamber over a $7.0 \times 8.7 \mathrm{~cm}$ opening through the wall of the animal containment area. The plate is secured to the main chamber by four screws. Atmospheric integrity is maintained by an O-ring seal in the plate that surrounds the opening in the main chamber. Integrity is maintained at the drinking spout by the fluid in the calibrated drinking tubes and spouts, effectively sealing the internal atmosphere.

The operant module is composed of the electrical and mechanical parts of a bar and reward-delivery system, modified from a Scientific Prototype operant box. The barpress unit was sealed in a $10.2 \times 8.8 \times 7.5 \mathrm{~cm}$ Plexiglas box, which can replace the drinking-tube plate. The bar passes through the $7.0 \times 8.7 \mathrm{~cm}$ opening into the animal containment area, thus providing easy access for the experimental animal. A reward-delivery unit, designed so that there is no intrusion into the main chamber, is located to the animal's left, $15 \mathrm{~cm}$ from the center of the bar. The barpress unit has its own supply of test atmosphere, so that atmospheric integrity is maintained within the chamber, and no dead spots are created by a stagnant atmosphere in the small, closed housing box. Atmospheric integrity also is maintained by the design of the reward-delivery module. A solenoid-driven fluid-delivery dipper travels down a vertical tube that is submerged in an open, external, liquid-reward holding tank (not pictured). Because the tube is not open to the external atmosphere (it is submerged in a fluid), intemal atmospheric integrity is preserved. When the bar is pressed, the dipper travels down the vertical tube, loads, and returns to an opening in the main chamber. (Only slight modification of the present designs would be required to allow the use of a solid-food-delivery unit with the chamber.) Again, the entire unit is held in place by four screws and is sealed by an O-ring seal as an integral part of the unit. When the chamber is not used for operant testing, the reward-delivery opening is sealed by a Plexiglas plate held in place by four screws. So that the 


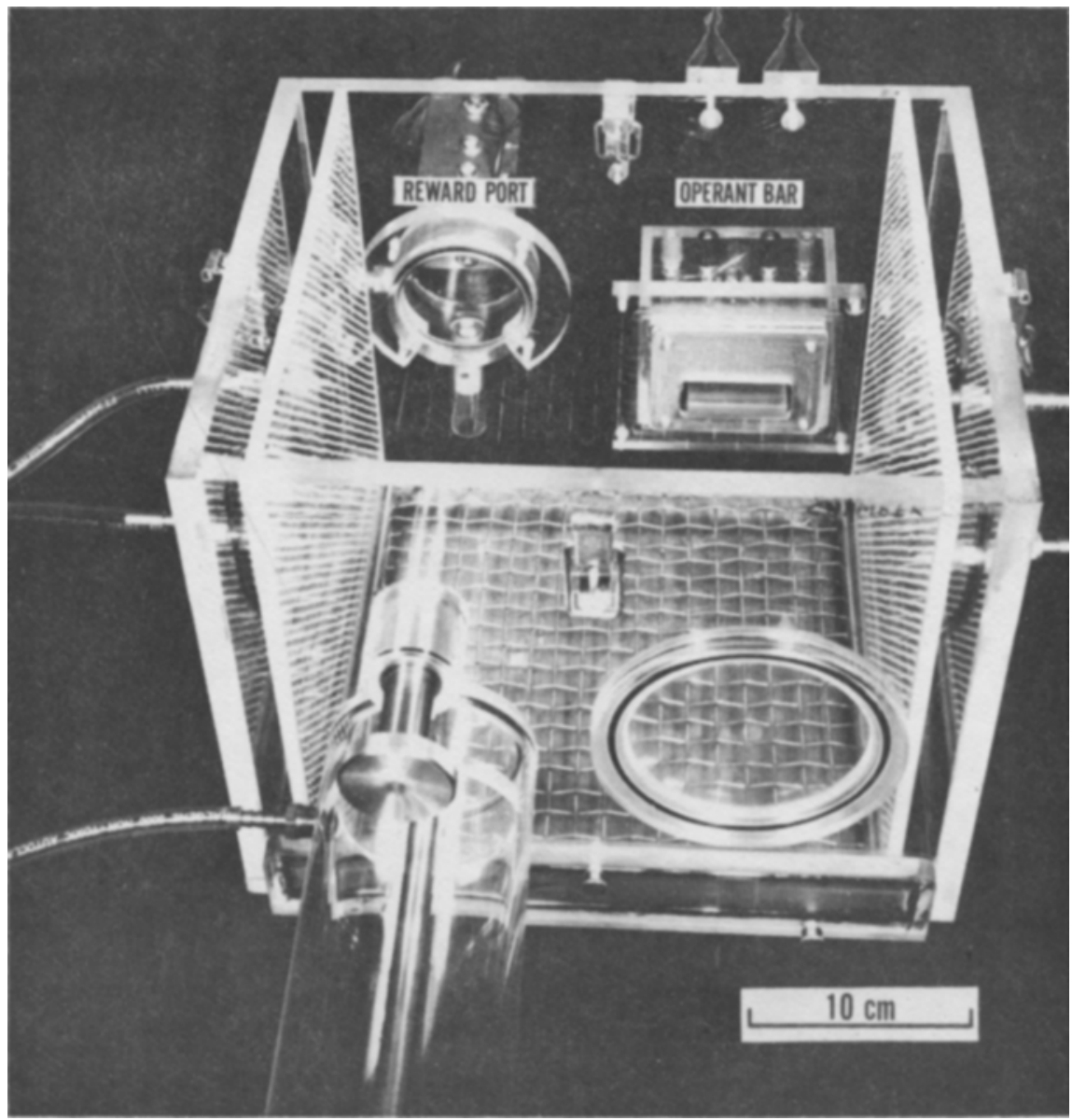

Figure 2. Top view of inhalation chamber, showing operant conditioning modules.

chamber may be used when there is no need for behavioral testing, the external modules can be replaced conveniently by Plexiglas plates.

\section{DISCUSSION}

The present chamber was designed to be used with rodents. When built to specifications, however, the chamber size (specifically, the animal loading capacity) is appropriate for most common laboratory animals. The 735-g upper weight limit would accommodate all small rodents, pigeons, and even small primates (e.g., marmosets, etc.). Furthermore, by expanding the atmospheric enclosure area, larger animals could be accommodated easily.

A major attraction of the chamber is its simple solution to an old problem of uniform diffusion. Additionally, the modular components provide a versatility of use in behavioral toxicology studies that has not been seen in the literature. A final attraction is low cost. The present chamber was built for less than $\$ 700$, including the cost of materials, labor, and behavioral attachments. This appears relatively inexpensive when compared with the $\$ 1,700$ cost reported by Rosenbaum et al. (1969) for a chronic-exposure chamber, a cost they said was less than that for systems available at that time for presenting nontoxic gases.

\section{REFERENCE NOTE}

1. Moss, O. R. Personal communication, 1980.

\section{REFERENCES}

Barrow, C. S., \& Dodd, D. E. Ammonia production in inhalation chambers and its relevance to chlorine inhalation studies. Toxicology and Applied Pharmacology, 1979, 49, 89-95.

Gounie, A. J., \& Dickens, D. W. Nitrous oxide-induced conditioned taste aversions in rats: The role of duration of drug exposure and its relation to the taste aversion-self-administration "paradox." Pharmacology, Biochemistry and Behavior, 1978, 9, 587-592.

Koblin, D. D., Dong, D. E., \& Eger, E. I. Tolerance of mice to nitrous oxide. Journal of Pharmacology and Experimental Therapeutics, 1979, 211, 317-325.

Leong, B. K. J., Powell, D. J., Pochyla, G. L., \& Lummis, M. G. An active dispersion inhalation exposure chamber. In B. K. J. Leong (Ed.), Inhalation toxicology and technology. Ann Arbor, Mich: Ann Arbor Science, 1981. 
MacFarland, H. N. Respiratory toxicology. In W. J. Hayes (Ed.), Essays in toxicology (Vol. 7). New York: Academic Press, 1976.

MacFarland, H. N., Ulrich, C. E., Martin, A., Krumm, A., Busey, W. M., \& Alarie, Y. Chronic exposure of cynomolgus monkeys to fly ash. In W. H. Walton (Ed.), Inhaled particles III (Vol. 1). Surrey, England: Unwin Brothers, Old Working, 1971.

Moss, O. R. Comparison of three methods of evaluating inhalation toxicology chamber performance. In B. K. J. Leong (Ed.), Inhalation toxicology and technology. Ann Arbor, Mich: Ann Arbor Science, 1981.

Rosenbaum, B. J., Makoff, D. L., \& Mills, L. J. A metabolic- environmental chamber for the study of unusual breathing atmospheres. Journal of Applied Physiology, 1969, 27, 892-894.

Wallenstein, M. C. The effect of nitrous oxide on time estimation in rats. Bulletin of the Psychonomic Society, 1976, 8, 118-120.

Walsh, J. M., \& Bachrach, A. J. Adaptation to nitrogen narcosis manifested by timing behavior in the rat. Journal of Comparative and Physiological Psychology, 1974, 85, 883-889.

(Manuscript received April 22, 1982;

revision accepted for publication April 19, 1983.) 